\title{
Determination of all non-normal quartic CM-fields and of all non-abelian normal octic CM-fields with class number one
}

\author{
by \\ Stéphane Louboutin (Caen) and Ryotaro Okazaki (Kyoto)
}

0. Introduction and notations. It has long been known that there are only finitely many non-isomorphic abelian CM-fields with class number one. Lately, K. Yamamura (see [Y]) has determined all abelian CM-fields with class number one. There are exactly 172 non-isomorphic such number fields. It is also known (see [O, Th. 2]) that there are only finitely many non-isomorphic normal CM-fields with class number one and J. Hoffstein proved that the degrees of such fields are less than 436 (see [H, Corollary $2]$ ). Hence, it is time to move on to the determination of non-abelian or even non-normal CM-fields with class number one and of fixed degrees. We look at the smallest possible degrees. We point out that there does not exist any non-abelian but normal CM-field with degree 6 . Hence, we will look at the non-normal quartic case and at the octic non-abelian normal case, i.e. at the quaternion or dihedral cases. Indeed, let $\boldsymbol{N}$ be a CM-field that is normal over $\mathbb{Q}$. As in [W, p. 38], one can easily see that the complex conjugation is an element of order two in the Galois group of the extension $N / \mathbb{Q}$ that commutes with all the other elements of this Galois group. This constraint on the Galois group of a normal CM-field enables us to point out that for example there does not exist any non-abelian but normal CM-field with degree $2 p$ where $p$ is an odd prime. Thus, the lowest possible degree for a non-abelian normal CM-field is 8 . Here we know that the Galois group of any non-abelian normal octic number field is either a quaternion group or a dihedral group.

In this paper, we prove that there does not exist any quaternion octic CM-field with class number one because the class number of such a number field is always even. Let us note that in [Lou 4] the first author determined all quaternion octic CM-fields with class number two. We then prove that there

1991 Mathematics Subject Classification: Primary 11R29; Secondary 11R11. 
are exactly 17 non-isomorphic dihedral octic CM-fields with class number one, and that there are exactly 37 non-isomorphic non-normal quartic CMfields with class number one.

These results rely on upper bounds for the discriminants of these number fields found by the first author in a previous paper (see [Lou 1]), and on a necessary condition for the relative class number of a CM-field to be one. This restricting necessary condition enables us to get the dihedral octic CMfields with relative class number one and the non-normal quartic CM-fields with relative class number one thanks to the numerical computation of the relative class numbers of only 26 non-normal quartic CM-fields. Finally, the computation of the class numbers of their maximal totally real subfields provides us with the ones with absolute class number one.

In the following five sections we proceed as follows. First, we show that normal quaternion octic CM-fields have even class numbers implying that there are no quaternion octic CM-fields with class number one. Second, we reduce the determination of the dihedral octic CM-fields with class number one to that of the ones with relative class number one. Thus, we describe the normal dihedral octic CM-fields with odd relative class numbers as a family $\left\{\boldsymbol{N}_{(p, q)}\right\}_{p, q}$ where $p$ and $q$ are primes subject to certain constraints. Third, we give a restricting necessary condition for the relative class number of a CM-field to be one. Fourth, thanks to this restricting necessary condition we determine the normal dihedral octic CM-fields with relative class number one: there are 19 non-isomorphic such fields. Fifth, thanks to the computation of the class numbers of their maximal totally real subfields we determine which dihedral octic CM-fields with relative class number one have class number one and determine the non-normal quartic CM-fields with class number one.

Notations. For any number field $\boldsymbol{F}$ we assume that $\boldsymbol{F}$ lies in the complex field and we set the following notations:

- $\boldsymbol{A}_{\boldsymbol{F}}$ is the ring of algebraic integers of $\boldsymbol{F}$,

- $\boldsymbol{E}_{\boldsymbol{F}}$ is the unit group of $\boldsymbol{F}$,

- $\boldsymbol{W}_{\boldsymbol{F}}$ is the group of roots of unity of $\boldsymbol{F}$,

- $H(\boldsymbol{F})$ is the ideal class group of $\boldsymbol{F}$,

- $h(\boldsymbol{F})$ is the class number of $\boldsymbol{F}$,

- $d(\boldsymbol{F})$ is the discriminant of $\boldsymbol{F}$.

For any CM-field $\boldsymbol{F}$ we set the following notations:

- $2 N=[\boldsymbol{F}: \mathbb{Q}]$ is the degree of $\boldsymbol{F}$,

- $\boldsymbol{F}_{+}$is the maximal totally real subfield of $\boldsymbol{F}$.

The complex conjugation is the non-trivial $\boldsymbol{F}_{+}$-isomorphism of the quadratic extension $\boldsymbol{F} / \boldsymbol{F}_{+}$. Set $\bar{\alpha}$ for the complex conjugate of $\alpha$. We remind 
the reader that in a CM-field every unit of absolute value 1 is a root of unity (see [W, p. 38]).

- $N=\left[\boldsymbol{F}_{+}: \mathbb{Q}\right]$ is the degree of $\boldsymbol{F}_{+}$,

- $d\left(\boldsymbol{F}_{+}\right)$is the discriminant of $\boldsymbol{F}_{+}$, so that $d\left(\boldsymbol{F}_{+}\right)^{2}$ divides $d(\boldsymbol{F})$,

- $\Delta(\boldsymbol{F})=\left|d(\boldsymbol{F}) / d\left(\boldsymbol{F}_{+}\right)\right|$, so that we have

$$
\Delta(\boldsymbol{F}) \geq d\left(\boldsymbol{F}_{+}\right) \quad \text { and } \quad \Delta(\boldsymbol{F}) \geq \sqrt{d(\boldsymbol{F})},
$$

- $Q_{\boldsymbol{F}} \in\{1,2\}$ is the Hasse unit index of $\boldsymbol{F}$ (see [W, Th. 4.12]),

- $h^{*}(\boldsymbol{F})=h(\boldsymbol{F}) / h\left(\boldsymbol{F}_{+}\right)$is the relative class number of $\boldsymbol{F}$,

- $H_{+}\left(\boldsymbol{F}_{+}\right)$is the strict ideal class group of $\boldsymbol{F}_{+}$,

- $h_{+}\left(\boldsymbol{F}_{+}\right)$is the strict class number of $\boldsymbol{F}_{+}$,

- $i_{\boldsymbol{F} / \boldsymbol{F}_{+}}$is the natural map from the fractional ideals of $\boldsymbol{F}_{+}$to those of $\boldsymbol{F}$.

1. Class numbers of non-abelian normal octic CM-fields. In this section we reduce the determination of normal octic CM-fields with class number one to that of non-normal quartic CM-fields with relative class number one. We firstly show that there are no quaternion octic CM-fields with class number one:

THEOREM 1. Relative class numbers of normal quaternion octic CMfields are even.

Let $\boldsymbol{N}$ be a normal octic CM-field with Galois group the quaternion group $\mathcal{Q}_{8}$ with eight elements: $\mathcal{Q}_{8}=\{ \pm 1, \pm i, \pm j, \pm k\}$ with $i^{2}=j^{2}=k^{2}=$ -1 and with $i j=k=-j i, j k=i=-k j$ and $k i=j=-i k$. Let $\boldsymbol{N}_{+}$be the totally real quartic subfield of $\boldsymbol{N}$. Then $\boldsymbol{N}_{+}$is bicyclic biquadratic. Let $\boldsymbol{k}_{0}, \boldsymbol{k}_{1}$ and $\boldsymbol{k}_{2}$ be the three quadratic subfields of $\boldsymbol{N}$, so that $\boldsymbol{N} / \boldsymbol{k}_{0}, \boldsymbol{N} / \boldsymbol{k}_{1}$ and $\boldsymbol{N} / \boldsymbol{k}_{2}$ are cyclic quartic. The lattice of subfields of $\boldsymbol{N}$ can be set out as follows:

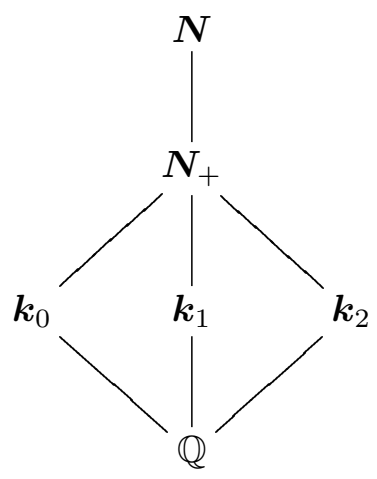

Now, as $\boldsymbol{N}_{+}$is totally real, $\boldsymbol{N}_{+}$cannot be $\mathbb{Q}(\sqrt{-1}, \sqrt{2})$ that is the only biquadratic bicyclic number field with exactly one finite ramified prime. 
Hence, at least two primes are ramified in the bicyclic biquadratic extension $\boldsymbol{N}_{+} / \mathbb{Q}$. Moreover, any prime ideal of $\boldsymbol{N}_{+}$that is ramified in $\boldsymbol{N}_{+} / \mathbb{Q}$ is also ramified in $\boldsymbol{N} / \boldsymbol{N}_{+}$. Indeed, it is ramified in at least one quadratic extension $\boldsymbol{N}_{+} / \boldsymbol{k}_{i}$. As $\boldsymbol{N} / \boldsymbol{k}_{i}$ is cyclic quartic, we see that it is totally ramified in $\boldsymbol{N} / \boldsymbol{k}_{i}$, hence ramified in $\boldsymbol{N} / \boldsymbol{N}_{+}$. We get Theorem 1 from the following Proposition 2 that will be used throughout this paper in order to prove that CM-fields $\boldsymbol{N}$ with odd relative class numbers are such that only few ideals of $\boldsymbol{N}_{+}$ramify in the quadratic extension $\boldsymbol{N} / \boldsymbol{N}_{+}$:

Proposition 2. Let $\boldsymbol{N}$ be a CM-field and let $\boldsymbol{N}_{+}$be its maximal totally real subfield. Then the relative class number of $\boldsymbol{N}$ is even provided that at least two prime ideals of $\boldsymbol{N}_{+}$ramify in the quadratic extension $\mathbf{N} / \boldsymbol{N}_{+}$.

Pro of. First, the kernel of $i_{\boldsymbol{N} / \boldsymbol{N}_{+}}$has order 1 or 2 (see [W, Th. 10.3]). Hence,

$$
\left|H(\boldsymbol{N}) / i_{\boldsymbol{N} / \boldsymbol{N}_{+}}\left(H\left(\boldsymbol{N}_{+}\right)\right)\right|= \begin{cases}h^{*}(\boldsymbol{N}) & \text { if } i_{\boldsymbol{N} / \boldsymbol{N}_{+}} \text {is injective, } \\ \frac{1}{2} h^{*}(\boldsymbol{N}) & \text { if } i_{\boldsymbol{N} / \boldsymbol{N}_{+}} \text {is not injective. }\end{cases}
$$

Second, let $\sigma$ denote the complex conjugation. If $\varepsilon$ is a unit of $N$ then $\bar{\varepsilon} / \varepsilon$ is a unit of absolute value 1 , hence a root of unity of $\boldsymbol{N}$, i.e. $\boldsymbol{W}_{\boldsymbol{N}}^{2} \subseteq \boldsymbol{U}_{\boldsymbol{N}}^{\sigma-1} \subseteq$ $\boldsymbol{W}_{\boldsymbol{N}}$. Now, let $t$ be the number of prime ideals $\boldsymbol{P}_{i}$ of $\boldsymbol{N}$ that are ramified in the quadratic extension $\boldsymbol{N} / \boldsymbol{N}_{+}$. We prove that $2^{t-1}$ divides $h^{*}(\boldsymbol{N})$. More precisely, we prove that if these $t$ prime ideals generate a subgroup of order $\leq 2^{t-1}$ in the group $H(\boldsymbol{N}) / i_{\boldsymbol{N} / \boldsymbol{N}_{+}}\left(H\left(\boldsymbol{N}_{+}\right)\right)$then they generate a subgroup of order $2^{t-1}$ and $i_{\boldsymbol{N} / \boldsymbol{N}_{+}}$is injective.

Indeed, let us suppose that there exists at least one non-empty and finite product $\boldsymbol{R}=\boldsymbol{P}_{i_{1}} \ldots \boldsymbol{P}_{i_{r}}$ of $r$ out of the $t$ prime ideals $\boldsymbol{P}_{i}$ 's such that its ideal class is in $i_{\boldsymbol{N} / \boldsymbol{N}_{+}}\left(H\left(\boldsymbol{N}_{+}\right)\right)$. Then there exists $\alpha \in \boldsymbol{N}$ and an integral ideal $\boldsymbol{I}$ of $\boldsymbol{N}_{+}$such that $\boldsymbol{R}=(\alpha) i_{\boldsymbol{N} / \boldsymbol{N}_{+}}(\boldsymbol{I})$. Then $(\bar{\alpha})=(\alpha)$, so that there exists a unit $\varepsilon$ in $\boldsymbol{N}$ such that $\bar{\alpha}=\varepsilon \alpha$. Then $\varepsilon$ is a unit of absolute value 1 , hence a root of unity of $\boldsymbol{N}$. If we had $\boldsymbol{W}_{\boldsymbol{N}}=\boldsymbol{U}_{\boldsymbol{N}}^{\sigma-1}$ then we would have $\varepsilon=\eta / \bar{\eta}$ for some unit $\eta$ in $\boldsymbol{N}$. Then $\beta_{+}=\eta \alpha$ is in $\boldsymbol{N}_{+}$and $\boldsymbol{R}=i_{\boldsymbol{N} / \boldsymbol{N}_{+}}\left(\left(\beta_{+}\right) \boldsymbol{I}\right)$ so that each prime ramified ideal $\boldsymbol{P}_{i}$ that divides $\boldsymbol{R}$ would divide $\boldsymbol{R}$ with an even power. A contradiction. Hence $\boldsymbol{U}_{\boldsymbol{N}}^{\sigma-1}=\boldsymbol{W}_{\boldsymbol{N}}^{2}, \varepsilon \in \boldsymbol{W}_{\boldsymbol{N}} \backslash \boldsymbol{W}_{\boldsymbol{N}}^{2}$ and we may assume that $\varepsilon=\zeta$ is a generator of the cyclic group $\boldsymbol{W}_{\boldsymbol{N}}$. Let $\beta \in \boldsymbol{A}_{\boldsymbol{N}}$ be such that $\zeta=\beta / \bar{\beta}$ (we may take $\beta=1+\zeta$ if $\zeta \neq-1$, and $\beta=\sqrt{-d}$ with $d \in \boldsymbol{A}_{\boldsymbol{N}_{+}}$such that $\boldsymbol{N}=\boldsymbol{N}_{+}(\sqrt{-d})$ if $\left.\zeta=-1\right)$. Then

$$
(\beta) \boldsymbol{R}=i_{\boldsymbol{N} / \boldsymbol{N}_{+}}\left(\left(\gamma_{+}\right) \boldsymbol{I}\right)
$$

where $\gamma_{+}=\alpha \beta$ is in $\boldsymbol{N}_{+}$. Hence, we have

$$
\boldsymbol{R}=\boldsymbol{R}_{\zeta} \stackrel{\text { def }}{=} \prod_{v_{\boldsymbol{P}_{i}}((\beta)) \text { odd }} \boldsymbol{P}_{i}
$$

where $\boldsymbol{R}_{\zeta}$ does not depend on $\beta$ such that $\zeta=\beta / \bar{\beta}$. Hence, there exists 
exactly one non-empty and finite product $\boldsymbol{R}=\boldsymbol{P}_{i_{1}} \cdot \ldots \cdot \boldsymbol{P}_{i_{r}}$ of $r$ out of the $t$ prime ideals $\boldsymbol{P}_{i}$ 's such that its ideal class is in $i_{\boldsymbol{N}} / \boldsymbol{N}_{+}\left(H\left(\boldsymbol{N}_{+}\right)\right)$. Thus, $2^{t-1}\left|i_{\boldsymbol{N} / \boldsymbol{N}_{+}}\left(H\left(\boldsymbol{N}_{+}\right)\right)\right|$divides $h(\boldsymbol{N})$. Moreover, $i_{\boldsymbol{N} / \boldsymbol{N}_{+}}$is injective. Indeed, if $\boldsymbol{J}$ were a non-principal ideal in $\boldsymbol{N}_{+}$such that $i_{\boldsymbol{N} / \boldsymbol{N}_{+}}(\boldsymbol{J})=\left(\alpha^{\prime}\right)$ then the same line of reasoning shows that

$$
(\beta) i_{\boldsymbol{N} / \boldsymbol{N}_{+}}(\boldsymbol{J})=i_{\boldsymbol{N} / \boldsymbol{N}_{+}}\left(\left(\gamma_{+}^{\prime}\right)\right)
$$

for some $\gamma_{+}^{\prime}$ in $\boldsymbol{N}_{+}$. Thus, $(*)$ and (**) would imply $\boldsymbol{R}=i_{\boldsymbol{N} / \boldsymbol{N}_{+}}\left(\left(\gamma_{+} / \gamma_{+}^{\prime}\right) \boldsymbol{I} \boldsymbol{J}\right)$ and each prime ramified ideal $\boldsymbol{P}_{i}$ that divides $\boldsymbol{R}$ would divide $\boldsymbol{R}$ with an even power. A contradiction. Hence, $2^{t-1}$ divides $h^{*}(\boldsymbol{N})$.

Lemma 3. Let $\boldsymbol{N}$ be a dihedral number field and let $\boldsymbol{K}$ be any non-normal quartic subfield of $\boldsymbol{N}$. Then $\boldsymbol{N}$ is a CM-field if and only if $\boldsymbol{K}$ is a CM-field (see [Lou 1]).

Let $\boldsymbol{N}$ be a dihedral octic CM-field. If $\boldsymbol{K}$ is a non-normal quartic subfield of $\boldsymbol{N}$ then

$$
h^{*}(\boldsymbol{N})=\frac{Q_{\boldsymbol{N}}}{2}\left(h^{*}(\boldsymbol{K})\right)^{2}
$$

(see [Lou 1]) with $Q_{\boldsymbol{N}} \in\{1,2\}$ being the Hasse unit index of $\boldsymbol{N}$.

We note that Theorem 1 and Lemma 3 reduce the determination of normal non-abelian octic CM-fields with relative class number one to that of non-normal quartic CM-fields with relative class number one.

2. Non-normal quartic CM-fields and dihedral octic CM-fields with odd relative class numbers. Let $\boldsymbol{K}$ be a non-normal quartic CMfield. Let $\boldsymbol{K}_{+}$be its real quadratic subfield and let $\delta$ be a totally positive element of $\boldsymbol{A}_{\boldsymbol{K}_{+}}$such that $\boldsymbol{K}=\boldsymbol{K}_{+}(\sqrt{-\delta})$. Then we have:

(1) The principal ideal $(\delta)$ of $\boldsymbol{A}_{\boldsymbol{K}_{+}}$is not a square in $\boldsymbol{A}_{\boldsymbol{K}_{+}}$so that $\boldsymbol{K} / \boldsymbol{K}_{+}$is ramified at at least one finite prime.

Proof. Indeed, if we had $(\delta)=\boldsymbol{I}^{2}$, then $N_{\boldsymbol{K}_{+} / \mathbf{Q}}(\delta)$ that is positive would be a square in $\mathbb{Q}$ so that $\boldsymbol{K} / \mathbb{Q}$ would be normal.

(2) The map $i_{\boldsymbol{K} / \boldsymbol{K}_{+}}$is injective from the ideal class group $H\left(\boldsymbol{K}_{+}\right)$of $\boldsymbol{K}_{+}$into the ideal class group $H(\boldsymbol{K})$ of $\boldsymbol{K}$.

Proof. Indeed, if $\boldsymbol{I}$ is a fractional ideal of $\boldsymbol{K}_{+}$such that $\boldsymbol{I} \boldsymbol{A}_{\boldsymbol{K}}=(\alpha)$ for some $\alpha \in \boldsymbol{K}$, then $(\bar{\alpha})=(\alpha)$ so that $\varepsilon=\bar{\alpha} / \alpha$ is a unit of $\boldsymbol{A}_{\boldsymbol{K}}$ of absolute value 1 , hence a root of unity of $\boldsymbol{K}$. Since $\boldsymbol{K} / \mathbb{Q}$ is non-normal, the group $\boldsymbol{W}_{\boldsymbol{K}}$ of roots of unity of $\boldsymbol{K}$ is $\{-1,+1\}$. Hence, either $\varepsilon=1$ and $\alpha$ is in $\boldsymbol{K}_{+}$ so that $\boldsymbol{I}$ is a principal fractional ideal of $\boldsymbol{K}_{+}$, or $\varepsilon=-1$ so that $\beta=\alpha \sqrt{-\delta}$ is in $\boldsymbol{K}_{+}$and $(\sqrt{-\delta}) \boldsymbol{I}=(\beta)$. Taking relative norms shows that the ideal $(\delta)$ of $\boldsymbol{K}_{+}$would be a square in $\boldsymbol{K}_{+}$, so that (1) provides us with the desired contradiction. 
From now on, we assume that $\boldsymbol{K}$ has odd relative class number $h^{*}(\boldsymbol{K})$. By [W, Th. 10.2], the class number $h\left(\boldsymbol{K}_{+}\right)$of the real quadratic subfield $\boldsymbol{K}_{+}$of $\boldsymbol{K}$ is odd. We now prove a little bit more:

(3) The strict class number $h_{+}\left(\boldsymbol{K}_{+}\right)$of $\boldsymbol{K}_{+}$is odd. Thus $\boldsymbol{K}_{+}=\mathbb{Q}(\sqrt{p})$ with $p \not \equiv 3(\bmod 4)$ a prime.

Pr o of. Let $\boldsymbol{L}$ be the class field over $\boldsymbol{K}_{+}$to the subgroup $H_{+}\left(\boldsymbol{K}_{+}\right)^{2}$ of $H_{+}\left(\boldsymbol{K}_{+}\right)$, the ideal class group of $\boldsymbol{K}_{+}$in the strict sense. Then $\boldsymbol{L} / \boldsymbol{K}_{+}$is not ramified at the finite places so that from (1) we get $\boldsymbol{L} \cap \boldsymbol{K}=\boldsymbol{K}_{+}$. Hence, $\boldsymbol{K} \boldsymbol{L} / \boldsymbol{K}$ is abelian and such that $\operatorname{Gal}(\boldsymbol{K} \boldsymbol{L} / \boldsymbol{K})$ is isomorphic to $\operatorname{Gal}\left(\boldsymbol{L} / \boldsymbol{K}_{+}\right)$ via restriction. Moreover, $\boldsymbol{K} \boldsymbol{L} / \boldsymbol{K}$ is unramified so that it is the class field over $\boldsymbol{K}$ to some subgroup $\boldsymbol{H}$ of $H(\boldsymbol{K})$. Hence, if $\operatorname{Fr}_{\boldsymbol{K} \boldsymbol{L} / \boldsymbol{K}}$ and $\operatorname{Fr}_{\boldsymbol{L} / \boldsymbol{K}_{+}}$denote the Artin maps, we have:

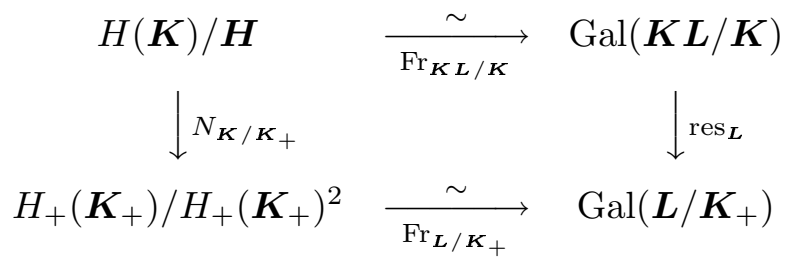

Since $N_{\boldsymbol{K} / \boldsymbol{K}_{+}} \circ i_{\boldsymbol{K} / \boldsymbol{K}_{+}}$is well defined from $H\left(\boldsymbol{K}_{+}\right)$to $H_{+}\left(\boldsymbol{K}_{+}\right)$and such that $N_{\boldsymbol{K} / \boldsymbol{K}_{+}} \circ i_{\boldsymbol{K} / \boldsymbol{K}_{+}}\left(H\left(\boldsymbol{K}_{+}\right)\right)$is included in $H_{+}\left(\boldsymbol{K}_{+}\right)^{2}$, we see that $i_{\boldsymbol{K} / \boldsymbol{K}_{+}}\left(H\left(\boldsymbol{K}_{+}\right)\right)$is included in the kernel of $\operatorname{Fr}_{\boldsymbol{K} \boldsymbol{L} / \boldsymbol{K}}$, i.e. in $\boldsymbol{H}$. Thus, by (2), the degree $[\boldsymbol{K} \boldsymbol{L}: \boldsymbol{K}]=\left[\boldsymbol{L}: \boldsymbol{K}_{+}\right]$divides $h^{*}(\boldsymbol{K})$, so that $\left[\boldsymbol{L}: \boldsymbol{K}_{+}\right]$is odd, and therefore the strict class number of $\boldsymbol{K}_{+}$is odd. Hence, we get the desired result.

(4) There is exactly one prime ideal $\boldsymbol{Q}_{+}$of $\boldsymbol{K}_{+}$that ramifies in $\boldsymbol{K} / \boldsymbol{K}_{+}$. Let $\boldsymbol{Q}$ denote the prime ideal of $\boldsymbol{N}$ lying above $\boldsymbol{Q}_{+}$. Then there exists a totally positive element $\delta_{+}$of $\boldsymbol{K}_{+}$such that $\boldsymbol{K}=\boldsymbol{K}_{+}\left(\sqrt{-\delta_{+}}\right),\left(\delta_{+}\right)=$ $\boldsymbol{Q}_{+}^{h\left(\boldsymbol{K}_{+}\right)}$and $\left(\sqrt{-\delta_{+}}\right)=\boldsymbol{Q}^{h\left(\boldsymbol{K}_{+}\right)}$.

P r o of. The first statement follows from Proposition 2 and (1). First, if a prime ideal $\boldsymbol{L}$ of $\boldsymbol{K}_{+}$is such that the exact power of $\boldsymbol{L}$ that divides the ideal $(\delta)$ is odd, then $\boldsymbol{L}$ is ramified in $\boldsymbol{K} / \boldsymbol{K}_{+}$. Second, the strict class number $h_{+}=h_{+}\left(\boldsymbol{K}_{+}\right)$of $\boldsymbol{K}_{+}$is odd and $h\left(\boldsymbol{K}_{+}\right)=h_{+}$. Now, noticing from (1) that the ideal $(\delta)$ is not a square in $\boldsymbol{K}_{+}$, we see that there exists a fractional ideal $\boldsymbol{I}$ of $\boldsymbol{K}_{+}$such that $(\delta)=\boldsymbol{Q}_{+} \boldsymbol{I}^{2}$. Raising this equality to the $h_{+}$-th power provides us with $\delta_{+}$and $x$ such that $\delta=\delta_{+} x^{2}$ where $\delta_{+}$is any totally positive generator of $\boldsymbol{Q}_{+}^{h_{+}}$. Hence, $\boldsymbol{K}=\boldsymbol{K}_{+}\left(\sqrt{-\delta_{+}}\right)$and $\left(\sqrt{-\delta_{+}}\right)^{2 h_{+}}=\boldsymbol{Q}^{2 h_{+}}$.

(5) Let $q$ be the rational prime lying below the prime ideal $\boldsymbol{Q}$ of (4). Then $q$ splits in $\boldsymbol{K}_{+} / \mathbb{Q}$ and $q \not \equiv 3(\bmod 4)$. Hence, if $p=2$ then $q \equiv 1$ $(\bmod 8)$. Moreover, $d(\boldsymbol{K})=q d\left(\boldsymbol{K}_{+}\right)^{2}$ provided that $q$ is odd. 
Proof. If $\boldsymbol{Q}_{+}$were inert in $\boldsymbol{K}_{+} / \mathbb{Q}$, then $N_{\boldsymbol{K}_{+} / \mathbb{Q}}\left(\delta_{+}\right)$that is positive would be a square in $\mathbb{Q}$ and as in (1) the extension $K / \mathbb{Q}$ would be normal. If $\boldsymbol{Q}_{+}$were ramified in $\boldsymbol{K}_{+} / \mathbb{Q}$, then by (3) we would have $\boldsymbol{Q}_{+}=(\sqrt{p})$ with $p$ the prime such that $\boldsymbol{K}_{+}=\mathbb{Q}(\sqrt{p})$. Hence, $N_{\boldsymbol{K}_{+} / \mathbf{Q}}\left(\delta_{+}\right)=p^{h_{+}}$would be a square in $\boldsymbol{K}_{+}=\mathbb{Q}(\sqrt{p})$ and $\boldsymbol{K} / \mathbb{Q}$ would be normal. Thus, we get the desired first result. For the second result, we first note that we may assume $q \neq 2$. Since $\boldsymbol{K} / \boldsymbol{K}_{+}=\boldsymbol{K} / \mathbb{Q}(\sqrt{p})$ is ramified only above $q$ (and at the infinite places), the normal closure $\boldsymbol{N}$ of $\boldsymbol{K}$ is such that $\boldsymbol{N} / \mathbb{Q}(\sqrt{p})$ is ramified only above $q$ (and at the infinite places). Since $\boldsymbol{N}$ contains $\mathbb{Q}(\sqrt{p}, \sqrt{q})$ (for we have $N_{\boldsymbol{K}_{+} / \mathbb{Q}}\left(\delta_{+}\right)=q^{h_{+}}$where $h_{+}$is odd $)$, we see that $\mathbb{Q}(\sqrt{p}, \sqrt{q}) / \mathbb{Q}(\sqrt{p})$ can be ramified only at the finite places above $q$, hence is unramified at the places above 2 , so that $q \not \equiv 3(\bmod 4)$. The last assertion follows from the fact that the quadratic extension $\boldsymbol{K} / \boldsymbol{K}_{+}$is ramified only at $\boldsymbol{Q}_{+}$, and this ideal is tamely ramified, so that the different $\mathcal{D}_{\boldsymbol{K} / \boldsymbol{K}_{+}}$of this quadratic extension is $\boldsymbol{Q}$ where $N_{\boldsymbol{K} / \mathbf{Q}}(\boldsymbol{Q})=N_{\boldsymbol{K}_{+} / \mathbf{Q}}\left(\boldsymbol{Q}_{+}\right)=q$.

(6) Let $\boldsymbol{N}$ be the normal closure of $\boldsymbol{K}$. Then $\boldsymbol{N}$ is a normal dihedral octic CM-field and its totally real quartic subfield $\boldsymbol{N}_{+}$is $\boldsymbol{N}_{+}=\mathbb{Q}(\sqrt{p}, \sqrt{q})$. Moreover, $\mathbf{N} / \mathbb{Q}(\sqrt{p q})$ is a cyclic quartic extension unramified at the finite places. Hence, 4 divides the strict class number of $\mathbb{Q}(\sqrt{p q})$ and 4 does not divide the class number of $\mathbb{Q}(\sqrt{p q})$.

P r o of. By the end of the proof of $(5), \mathbb{Q}(\sqrt{p})$ and $\mathbb{Q}(\sqrt{q})$ are quadratic subfields of $\boldsymbol{N}$. Hence, $\mathbb{Q}(\sqrt{p q})$ is the third quadratic subfield of the dihedral octic field $\boldsymbol{N}$ and $\boldsymbol{N}_{+}=\mathbb{Q}(\sqrt{p}, \sqrt{q})$ is a totally real quartic subfield of $\boldsymbol{N}$. Since $\boldsymbol{K}$ is totally imaginary, so is $\boldsymbol{N}$. Hence, $\boldsymbol{N}$ is a CM-field. Let $\widetilde{\boldsymbol{K}}$ be one of the two non-normal quartic subfields of $\boldsymbol{N}$ that are not isomorphic to $\boldsymbol{K}$, so that $\widetilde{\boldsymbol{K}}$ is not a quadratic extension of $\mathbb{Q}(\sqrt{p})$. As $h^{*}(\widetilde{\boldsymbol{K}})=h^{*}(\boldsymbol{K})$ is odd, we see from $(3)$ that $\widetilde{\boldsymbol{K}}$ is a quadratic extension of $\mathbb{Q}(\sqrt{q})$. As $\boldsymbol{K} / \mathbb{Q}(\sqrt{p})$ is unramified above $p$ and $\boldsymbol{N} / \mathbb{Q}(\sqrt{p})$ is the normal closure of $\boldsymbol{K} / \mathbb{Q}(\sqrt{p})$ it follows that $\mathbf{N} / \mathbb{Q}(\sqrt{p})$ is unramified above $p$. In the same way, $\mathbf{N} / \mathbb{Q}(\sqrt{q})$ is unramified above $q$. Computing ramification indices in the tower shows that $p$ and $q$ have ramification indices equal to 2 in $\boldsymbol{N} / \mathbb{Q}$. Hence, $\boldsymbol{N} / \mathbb{Q}(\sqrt{p q})$ is unramified at the finite places. The assertions concerning the class number of $\mathbb{Q}(\sqrt{p q})$ follow from class field theory and from the fact that the 2-Sylow subgroups of the ideal class group of $\mathbb{Q}(\sqrt{p q})$ are cyclic in the wide and narrow senses provided that $p$ and $q$ are distinct primes not congruent to 3 $(\bmod 4)$.

(7) Conversely, we have:

Theorem/Definition. Let $p$ and $q$ be two distinct primes that are not congruent to $3(\bmod 4)$ and such that $q$ splits in $\mathbb{Q}(\sqrt{p})$. Let $\boldsymbol{N}_{(p, q)}$ denote the unique cyclic quartic extension of $\mathbb{Q}(\sqrt{p q})$ unramified at the finite places 
(hence $\left.\boldsymbol{N}_{(p, q)}=\boldsymbol{N}_{(q, p)}\right)$. Then $\boldsymbol{N}_{(p, q)}$ is a dihedral octic field. Moreover, let $\boldsymbol{K}_{(p, q)}$ denote any one of the two isomorphic non-normal quartic subfields of $\boldsymbol{N}_{(p, q)}$ that contain the real quadratic field $\mathbb{Q}(\sqrt{p})$. The following three conditions are equivalent:

(a) $\boldsymbol{N}_{(p, q)}$ is a CM-field,

(b) $\boldsymbol{K}_{(p, q)}$ is a CM-field,

(c) 4 does not divide the class number of the real quadratic field $\mathbb{Q}(\sqrt{p q})$ (let us point out that this implies $N_{\mathbf{Q}(\sqrt{p q}) / \mathbf{Q}}\left(\varepsilon_{p q}\right)=+1$ where $\varepsilon_{p q}>1$ is the fundamental unit of the real quadratic field $\mathbb{Q}(\sqrt{p q}))$.

Proof. As $q$ splits in $\mathbb{Q}(\sqrt{p})$, it follows that 4 divides the strict class number of $\mathbb{Q}(\sqrt{p q})$ and 2 divides the class number of $\mathbb{Q}(\sqrt{p q})$. Moreover, the 2-Sylow subgroups of the narrow ideal class group and of the wide ideal class group of $\mathbb{Q}(\sqrt{p q})$ are cyclic. Let $2^{n_{+}}, n_{+} \geq 2$, and $2^{n}, n \geq 1$, be the orders of these 2-Sylow subgroups in the narrow sense and in the wide sense (so that $n_{+}=n$ or $n_{+}=n+1$ ). Let $\boldsymbol{H}_{(p, q)}$ be the Hilbert 2-class field of $\mathbb{Q}(\sqrt{p q})$, so $\boldsymbol{H}_{(p, q)}$ is the maximal unramified abelian 2-extension of $\mathbb{Q}(\sqrt{p q})$ and $\operatorname{Gal}\left(\boldsymbol{H}_{(p, q)} / \mathbb{Q}(\sqrt{p q})\right)$ is isomorphic to the 2-Sylow subgroup of the ideal class group of $\mathbb{Q}(\sqrt{p q})$. Let $\boldsymbol{H}_{(p, q)}^{+}$be the strict Hilbert 2-class field of $\mathbb{Q}(\sqrt{p q})$, so $\boldsymbol{H}_{(p, q)}^{+}$is the maximal abelian 2-extension of $\mathbb{Q}(\sqrt{p q})$ unramified at the finite places and $\operatorname{Gal}\left(\boldsymbol{H}_{(p, q)}^{+} / \mathbb{Q}(\sqrt{p q})\right)$ is isomorphic to the 2-Sylow subgroup of the narrow ideal class group of $\mathbb{Q}(\sqrt{p q})$. Then $\boldsymbol{H}_{(p, q)} \subseteq \boldsymbol{H}_{(p, q)}^{+}$, and $\boldsymbol{H}_{(p, q)}^{+} / \mathbb{Q}(\sqrt{p q})$ is cyclic of degree $2^{n_{+}}$and $\boldsymbol{H}_{(p, q)} / \mathbb{Q}(\sqrt{p q})$ is cyclic of degree $2^{n}$. Moreover, the maximality of $\boldsymbol{H}_{(p, q)}$ implies that $\boldsymbol{H}_{(p, q)} / \mathbb{Q}$ is normal, hence $\boldsymbol{H}_{(p, q)}$ contains a normal quartic number field that is an unramified quadratic extension of $\mathbb{Q}(\sqrt{p q})$, hence contains $\mathbb{Q}(\sqrt{p}, \sqrt{q})$. Hence, we have

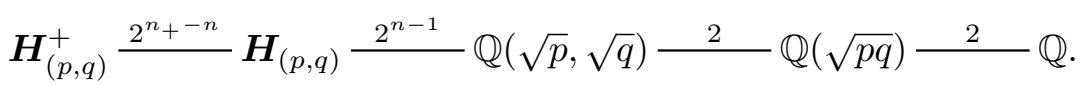

In the same way, $\boldsymbol{H}_{(p, q)}^{+} / \mathbb{Q}$ is normal, hence $\boldsymbol{H}_{(p, q)}^{+}$is either totally real or totally imaginary. The maximality of $\boldsymbol{H}_{(p, q)}$ implies that $\boldsymbol{H}_{(p, q)}^{+}$is totally real if and only if $\boldsymbol{H}_{(p, q)}^{+}=\boldsymbol{H}_{(p, q)}$, hence if and only if $n_{+}=n$. Moreover, if $\boldsymbol{H}_{(p, q)}^{+}$is totally imaginary, then it is a quadratic extension of the totally real number field $\boldsymbol{H}_{(p, q)}$, hence $\boldsymbol{H}_{(p, q)}^{+}$is a CM-field.

Now, by class field theory, there exists a unique cyclic quartic extension $\boldsymbol{N}_{(p, q)}$ of $\mathbb{Q}(\sqrt{p q})$ unramified at the finite places, namely the only subfield of $\boldsymbol{H}_{(p, q)}^{+}$that is a quartic extension of $\mathbb{Q}(\sqrt{p q})$. Since $\boldsymbol{H}_{(p, q)}^{+} / \mathbb{Q}$ is normal and $\boldsymbol{H}_{(p, q)}^{+} / \boldsymbol{N}_{(p, q)}$ is cyclic, $\boldsymbol{N}_{(p, q)} / \mathbb{Q}$ is normal. Moreover, it is not abelian since the genus number field of $\mathbb{Q}(\sqrt{p q})$ is $\mathbb{Q}(\sqrt{p}, \sqrt{q})$. Thus, it is dihedral or quaternionic. In fact, $\boldsymbol{N}_{(p, q)}$ is a dihedral octic field. Indeed, it suffices to note 
that the quartic extension $\boldsymbol{N}_{(p, q)} / \mathbb{Q}(\sqrt{p})$ is not cyclic since $q$ that ramifies in the quadratic extension $\mathbb{Q}(\sqrt{p}, \sqrt{q}) / \mathbb{Q}(\sqrt{p})$ would be totally ramified in the cyclic quartic extension $\boldsymbol{N}_{(p, q)} / \mathbb{Q}(\sqrt{p})$, hence ramified in the quadratic extension $\boldsymbol{N}_{(p, q)} / \mathbb{Q}(\sqrt{p}, \sqrt{q})$.

Now we prove that $(\mathrm{a}) \Leftrightarrow(\mathrm{c})$. If $n \geq 2$ then $\boldsymbol{N}_{(p, q)}$ is included in $\boldsymbol{H}_{(p, q)}$, hence $\boldsymbol{N}_{(p, q)}$ is totally real. If $n=1$, then $n_{+}=2$ and $\boldsymbol{H}_{(p, q)}=\mathbb{Q}(\sqrt{p}, \sqrt{q})$ and $\boldsymbol{N}_{(p, q)}=\boldsymbol{H}_{(p, q)}^{+}$is a CM-field. Finally, (a) $\Leftrightarrow(\mathrm{b})$ was proved in [Lou 1] (see Lemma 3).

(8) If $\boldsymbol{K}_{(p, q)}$ is a CM-field then $h^{*}\left(\boldsymbol{K}_{(p, q)}\right)$ is odd.

Proof. We set $\boldsymbol{K}=\boldsymbol{K}_{(p, q)}, \boldsymbol{K}_{+}=\boldsymbol{k}_{p}=\mathbb{Q}(\sqrt{p})$ and let $\varepsilon_{+}$be the fundamental unit of $\boldsymbol{K}_{+}$, so that $N_{\boldsymbol{K}_{+} / \mathbb{Q}}\left(\varepsilon_{+}\right)=-1$. Since $Q_{\boldsymbol{K}}=1$ and $\boldsymbol{W}_{\boldsymbol{K}}=\{-1,+1\}$, we have $\boldsymbol{E}_{\boldsymbol{K}}=\boldsymbol{E}_{\boldsymbol{K}_{+}}$. Now, let $\mathcal{C}$ be an ideal class of order $\leq 2$ in the ideal class group of $\boldsymbol{K}$. Since $N_{\boldsymbol{K} / \boldsymbol{K}_{+}}(\mathcal{C})$ has order $\leq 2$ in the ideal class group of $\boldsymbol{K}_{+}$that has odd order, it follows that $N_{\boldsymbol{K} / \boldsymbol{K}_{+}}(\mathcal{C})$ is principal, so that $\mathcal{C} \overline{\mathcal{C}}=i_{\boldsymbol{K} / \boldsymbol{K}_{+}}\left(N_{\boldsymbol{K} / \mathbf{Q}}(\mathcal{C})\right)$ is principal, and thus $\overline{\mathcal{C}}=\mathcal{C}$, i.e. $\mathcal{C}$ is an ambiguous class. Let $\boldsymbol{I}$ be an integral ideal in $\mathcal{C}$. There exists $\alpha$ in $\boldsymbol{K}$ such that $\overline{\boldsymbol{I}}=(\alpha) \boldsymbol{I}$. Hence, $(\alpha \bar{\alpha})=(1)$ so that there exists an integer $n$ such that $\alpha \bar{\alpha}=\varepsilon_{+}^{2 n}$ (indeed, $\alpha \bar{\alpha}$ is positive and in $\boldsymbol{E}_{\boldsymbol{K}}=\boldsymbol{E}_{\boldsymbol{K}_{+}}$so that there exists an integer $m$ such that $\alpha \bar{\alpha}=\varepsilon_{+}^{m}$; since $0<N_{\boldsymbol{K} / \mathbf{Q}}(\alpha)=(-1)^{m}, m$ is even). If $\beta=\alpha / \varepsilon_{+}^{n}$ then $\overline{\boldsymbol{I}}=(\beta) \boldsymbol{I}$ and $\beta \bar{\beta}=1$ so that there exists an algebraic integer $\gamma$ in $\boldsymbol{K}$ such that $\beta=\gamma / \bar{\gamma}$. Thus, $\overline{\boldsymbol{J}}=\boldsymbol{J}$ where $\boldsymbol{J}=(\gamma) \boldsymbol{I}$ is in $\mathcal{C}$. It follows that there exists an integral ideal $\boldsymbol{L}$ of $\boldsymbol{K}_{+}$such that $\boldsymbol{J}=i_{\boldsymbol{K} / \boldsymbol{K}_{+}}(\boldsymbol{L})$ or $\boldsymbol{J}=i_{\boldsymbol{K} / \boldsymbol{K}_{+}}(\boldsymbol{L}) \boldsymbol{Q}$ where $\boldsymbol{Q}$ is the prime ideal of $\boldsymbol{K}$ ramified in $\boldsymbol{K} / \boldsymbol{K}_{+}$ given in (4). By (4), $\boldsymbol{J}^{h\left(\boldsymbol{K}_{+}\right)}$is principal so that the order of $\mathcal{C}$ is odd, i.e. $\mathcal{C}$ is the principal ideal class. Hence, there does not exist an ideal class of order 2 , so that $h(\boldsymbol{K})$ is odd.

Hence, we have proved:

THEOREM 4. A number field is a non-normal quartic CM-field with odd relative class number if and only if it is a subfield of a dihedral octic CM-field $\boldsymbol{N}_{(p, q)}$ that is a cyclic quartic extension of the real quadratic field $\mathbb{Q}(\sqrt{p q})$, unramified at the finite places, such that 4 does not divide the class number of $\mathbb{Q}(\sqrt{p q})$, where $p$ and $q$ are distinct primes not congruent to $3(\bmod 4)$ and $q$ splits in $\mathbb{Q}(\sqrt{p})$.

We finish this section by giving an upper bound on discriminants of non-normal quartic CM-fields with relative class number one:

TheOREM 5 (see [Lou 1]). Let $\boldsymbol{K}$ be a non-normal quartic CM-field, and let $\boldsymbol{k}$ be its real quadratic subfield. Then $\boldsymbol{K}$ has relative class number greater 
than one provided that

$$
\Delta(\boldsymbol{K}) \stackrel{\text { def }}{=} \frac{d(\boldsymbol{K})}{d(\boldsymbol{k})} \geq 5 \cdot 10^{9} .
$$

Remark.

$($ see $(5))$.

$$
\Delta\left(\boldsymbol{K}_{(p, q)}\right)= \begin{cases}p q & \text { if } 2 \neq p<q \\ 8 q & \text { if } 2=p<q\end{cases}
$$

3. A criterion for the relative class number of a CM-field to be one. We show that CM-fields with relative class number one have the remarkable property that prime ideals that split completely have large absolute norm:

TheOREM 6. Let $\boldsymbol{F}$ be a CM-field, and assume that the natural map $i_{\boldsymbol{F} / \boldsymbol{F}_{+}}$from the group of fractional ideals of $\boldsymbol{F}_{+}$to that of $\boldsymbol{F}$ is injective. Assume that $h^{*}(\boldsymbol{F})=1$ and let $\boldsymbol{P}_{+}$be a prime ideal of $\boldsymbol{F}_{+}$that is not inert in $\boldsymbol{F} / \boldsymbol{F}_{+}$. Then

$$
N_{\boldsymbol{F}_{+} / \mathbf{Q}}\left(\boldsymbol{P}_{+}\right) \geq \frac{1}{4^{N}} \frac{d(\boldsymbol{F})}{d\left(\boldsymbol{F}_{+}\right)^{2}} .
$$

Proof. Let $\boldsymbol{P}$ be a prime ideal of $\boldsymbol{F}$ lying above $\boldsymbol{P}_{+}$. Since $h^{*}(\boldsymbol{F})=1$ and since $i_{\boldsymbol{F} / \boldsymbol{F}_{+}}$is injective, we have

$$
H(\boldsymbol{F})=i_{\boldsymbol{F} / \boldsymbol{F}_{+}}\left(H\left(\boldsymbol{F}_{+}\right)\right) .
$$

Hence, there exists an ideal $\boldsymbol{I}$ of $\boldsymbol{F}_{+}$such that $\boldsymbol{I} \boldsymbol{P}$ is principal in $\boldsymbol{F}$, i.e. there exists $\alpha \in \boldsymbol{A}_{\boldsymbol{F}}$ such that $\boldsymbol{I P}=(\alpha)$. Let us point out that since the prime ideal $\boldsymbol{P}$ is not inert in $\boldsymbol{F} / \boldsymbol{F}_{+}$, we have $\alpha \in \boldsymbol{F} \backslash \boldsymbol{F}_{+}$, so that $\bar{\alpha}-\alpha \neq 0$. Taking norms, we get

$$
N_{\boldsymbol{F}_{+} / \mathbf{Q}}\left(\boldsymbol{P}_{+}\right) N_{\boldsymbol{F}_{+} / \mathbf{Q}}(\boldsymbol{I})^{2}=N_{\boldsymbol{F} / \mathbf{Q}}(\boldsymbol{I} \boldsymbol{P})=N_{\boldsymbol{F}_{+} / \mathbf{Q}}\left(N_{\boldsymbol{F} / \boldsymbol{F}_{+}}(\alpha)\right) .
$$

Now,

$$
N_{\boldsymbol{F} / \boldsymbol{F}_{+}}(\alpha)=\bar{\alpha} \alpha=\frac{\beta+\gamma}{4}
$$

with $\beta=(\bar{\alpha}+\alpha)^{2}=N_{\boldsymbol{F} / \boldsymbol{F}_{+}}(\bar{\alpha}+\alpha)$ and $\gamma=-(\bar{\alpha}-\alpha)^{2}=N_{\boldsymbol{F} / \boldsymbol{F}_{+}}(\bar{\alpha}-\alpha)$.

Since $\beta$ and $\gamma$ are totally positive elements in $\boldsymbol{F}_{+}$, we get

$$
\begin{aligned}
N_{\boldsymbol{F}_{+} / \mathbf{Q}}\left(\boldsymbol{P}_{+}\right) N_{\boldsymbol{F}_{+} / \mathbf{Q}}(\boldsymbol{I})^{2} & =\frac{1}{4^{N}} N_{\boldsymbol{F}_{+} / \mathbf{Q}}(\beta+\gamma) \\
& \geq \frac{1}{4^{N}} N_{\boldsymbol{F}_{+} / \mathbf{Q}}(\gamma)=\frac{1}{4^{N}} N_{\boldsymbol{F} / \mathbf{Q}}(\bar{\alpha}-\alpha) .
\end{aligned}
$$

Let $\mathcal{D}_{\boldsymbol{F} / \boldsymbol{F}_{+}}$be the different of the quadratic extension $\boldsymbol{F} / \boldsymbol{F}_{+}$. We have

$$
N_{\boldsymbol{F} / \mathbf{Q}}\left(\boldsymbol{I} \mathcal{D}_{\boldsymbol{F} / \boldsymbol{F}_{+}}\right)=N_{\boldsymbol{F}_{+} / \mathbf{Q}}(\boldsymbol{I})^{2} \frac{d(\boldsymbol{F})}{d\left(\boldsymbol{F}_{+}\right)^{2}} .
$$


Hence, it suffices to prove that the ideal $\boldsymbol{I D}_{\boldsymbol{F} / \boldsymbol{F}_{+}}$divides the principal ideal $(\bar{\alpha}-\alpha)$. Thus, we prove that for any prime ideal $\boldsymbol{L}$ of $\boldsymbol{F}$ we have

$$
v_{\boldsymbol{L}}(\bar{\alpha}-\alpha) \geq v_{\boldsymbol{L}}\left(\boldsymbol{I} \boldsymbol{A}_{\boldsymbol{F}}\right)+v_{\boldsymbol{L}}\left(\mathcal{D}_{\boldsymbol{F} / \boldsymbol{F}_{+}}\right) .
$$

First, if $\boldsymbol{L}$ is not ramified in $\boldsymbol{F} / \boldsymbol{F}_{+}$, then $v_{\boldsymbol{L}}\left(\mathcal{D}_{\boldsymbol{F} / \boldsymbol{F}_{+}}\right)=0$ and $(*)$ is clearly satisfied since $\alpha \in \boldsymbol{I} \boldsymbol{A}_{F}$ and $\bar{\alpha} \in \boldsymbol{I} \boldsymbol{A}_{\boldsymbol{F}}$. Second, if $\boldsymbol{L}$ is ramified in $\boldsymbol{F} / \boldsymbol{F}_{+}$and if $\boldsymbol{G}_{i}, i \geq-1$, are the ramification subgroups of the Galois group $\boldsymbol{G}=\{\mathrm{Id}, \sigma\}$ (where $\sigma$ is the complex conjugation) of the quadratic extension $\boldsymbol{F} / \boldsymbol{F}_{+}$, then we have (see [Se, Chapitre IV, Proposition 4])

$$
v_{\boldsymbol{L}}\left(\mathcal{D}_{\boldsymbol{F} / \boldsymbol{F}_{+}}\right)=\sum_{i \geq 0}\left(\operatorname{Card}\left(\boldsymbol{G}_{i}\right)-1\right)=1+\max \left\{i: i \geq 0 \text { and } \sigma \in \boldsymbol{G}_{i}\right\} .
$$

Now, $v_{\boldsymbol{L}}(\bar{\alpha}-\alpha) \geq a+i+1$ if $\sigma \in \boldsymbol{G}_{i}$ and if $v_{\boldsymbol{L}}(\alpha) \geq a$, hence if $v_{\boldsymbol{L}}\left(\boldsymbol{I} \boldsymbol{A}_{\boldsymbol{F}}\right) \geq a$. Hence, we get the desired result.

4. Determination of the non-normal quartic CM-fields and of the dihedral octic CM-fields with relative class number one. Let us recall the following rules:

Lemma. Let $\boldsymbol{N} / \boldsymbol{F}$ be a bicyclic biquadratic extension of number fields. Let $\boldsymbol{K}_{i} / \boldsymbol{F}, 0 \leq i \leq 2$, be the three quadratic subextensions of $\boldsymbol{N} / \boldsymbol{F}$. Let $\boldsymbol{P}_{\boldsymbol{F}}$ be a prime ideal of $\boldsymbol{A}_{\boldsymbol{F}}$ that is not ramified in $\boldsymbol{N} / \boldsymbol{F}$. If $\boldsymbol{P}_{\boldsymbol{F}}$ is inert in $\boldsymbol{K}_{1} / \boldsymbol{F}$ and in $\boldsymbol{K}_{2} / \boldsymbol{F}$, then $\boldsymbol{P}_{\boldsymbol{F}}$ splits in $\boldsymbol{K}_{0} / \boldsymbol{F}$ and the prime ideals of $\boldsymbol{K}_{0}$ lying above $\boldsymbol{P}_{\boldsymbol{F}}$ are inert in $\boldsymbol{N} / \boldsymbol{K}_{0}$.

Let $\boldsymbol{N}$ be a dihedral octic number field. Let $\boldsymbol{K}_{1}, \boldsymbol{K}_{1}^{\prime}, \boldsymbol{K}_{2}$ and $\boldsymbol{K}_{2}^{\prime}$ be the non-normal quartic subfields of $\boldsymbol{N}$, where $\boldsymbol{K}_{1}^{\prime}$ is isomorphic to $\boldsymbol{K}_{1}, \boldsymbol{K}_{2}^{\prime}$ is isomorphic to $\boldsymbol{K}_{2}$ and $\boldsymbol{K}_{1}$ is not isomorphic to $\boldsymbol{K}_{2}$. Let $\boldsymbol{k}_{i}$ be the quadratic subfield of $\boldsymbol{K}_{i}$, so that each $\boldsymbol{N} / \boldsymbol{k}_{i}$ is a bicyclic biquadratic extension. Let $\boldsymbol{k}_{+}$be the quadratic subfield of the only quartic subfield $\boldsymbol{N}_{+}$of $\boldsymbol{N}$ that is normal over $\mathbb{Q}$, so that $\boldsymbol{N}_{+} / \mathbb{Q}$ is bicyclic biquadratic. This situation can be well understood with the help of the following lattice of subfields:

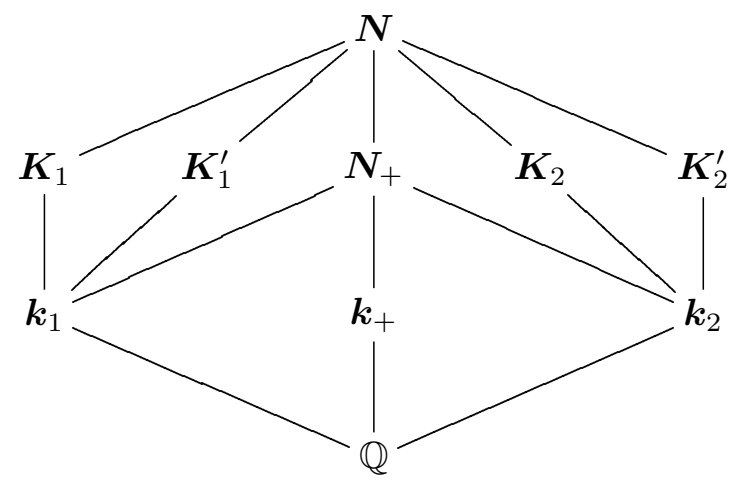


Now, let $l$ be a prime number that is unramified in $\boldsymbol{N} / \mathbb{Q}$, let $\boldsymbol{L}_{1}$ be a prime ideal of $\boldsymbol{k}_{1}$ lying above $l$, let $\boldsymbol{L}_{+}$be any prime ideal of $\boldsymbol{N}_{+}$lying above $\boldsymbol{L}_{1}$ and let $\boldsymbol{L}_{2}$ be the prime ideal of $\boldsymbol{k}_{2}$ lying below $\boldsymbol{L}_{+}$. Let us assume that $\boldsymbol{L}_{1}$ is inert in $\boldsymbol{K}_{1} / \boldsymbol{k}_{1}$ and in $\boldsymbol{K}_{1}^{\prime} / \boldsymbol{k}_{1}$. We prove that $l$ splits in $\boldsymbol{k}_{+} / \mathbb{Q}$.

Indeed, from the previous lemma, $\boldsymbol{L}_{1}$ that is inert in $\boldsymbol{K}_{1} / \boldsymbol{k}_{1}$ and in $\boldsymbol{K}_{1}^{\prime} / \boldsymbol{k}_{1}$ splits completely in $\boldsymbol{N}_{+} / \boldsymbol{k}_{1}$ and the prime ideals of $\boldsymbol{N}_{+}$lying above $\boldsymbol{L}_{1}$ are inert in $\boldsymbol{N} / \boldsymbol{N}_{+}$. Hence, $\boldsymbol{L}_{2}$ splits in $\boldsymbol{N}_{+} / \boldsymbol{k}_{2}$ for if it were inert then it would be inert in $\boldsymbol{N} / \boldsymbol{k}_{2}$, hence in $\boldsymbol{K}_{2} / \boldsymbol{k}_{2}$ and in $\boldsymbol{K}_{2}^{\prime} / \boldsymbol{k}_{2}$, so that the previous lemma would tell us that it would split in $\boldsymbol{N}_{+} / \boldsymbol{k}_{2}$. Hence, we have just proved that $\boldsymbol{L}_{+}$splits in $\boldsymbol{N}_{+} / \boldsymbol{k}_{1}$ and in $\boldsymbol{N}_{+} / \boldsymbol{k}_{2}$. We deduce that $l$ splits in $\boldsymbol{k}_{+} / \mathbb{Q}$ for if it were inert then it would be inert in $\boldsymbol{k}_{1} / \mathbb{Q}$ and in $\boldsymbol{k}_{2} / \mathbb{Q}$, and the previous lemma would provide us with a contradiction.

Now, let $\boldsymbol{N}$ be a dihedral octic CM-field with relative class number one. As $\boldsymbol{N} / \boldsymbol{N}_{+}$is unramified at the finite places, we get $d(\boldsymbol{N})=d\left(\boldsymbol{N}_{+}\right)^{2}$ so that Theorem 6 is useless. Nevertheless, by Lemma 3, the non-normal quartic CM-subfields $\boldsymbol{K}_{1}$ and $\boldsymbol{K}_{1}^{\prime}$ of $\boldsymbol{N}$ also have relative class number one. By Theorem 6 , any prime ideal $\boldsymbol{L}_{1}$ of $\boldsymbol{k}_{1}$ remains inert in $\boldsymbol{K}_{1} / \boldsymbol{k}_{1}$ and in $\boldsymbol{K}_{1}^{\prime} / \boldsymbol{k}_{1}$ provided that its norm is less than $d\left(\boldsymbol{K}_{1}\right) / 16 d\left(\boldsymbol{k}_{1}\right)^{2}=d\left(\boldsymbol{K}_{1}^{\prime}\right) / 16 d\left(\boldsymbol{k}_{1}\right)^{2}$. Hence, if $\boldsymbol{N}=\boldsymbol{N}_{(p, q)}$ has relative class number one, then so does $\boldsymbol{K}_{1}=\boldsymbol{K}_{(p, q)}$. Hence any prime ideal $\boldsymbol{L}$ of $\mathbb{Q}(\sqrt{p})$ such that

$$
N_{\boldsymbol{k}_{p} / \mathbf{Q}}(\boldsymbol{L})<\frac{1}{16} \frac{d\left(\boldsymbol{K}_{(p, q)}\right)}{d\left(\boldsymbol{k}_{p}\right)^{2}}=\frac{q}{16}
$$

(see (5) for the equality) is inert in $\boldsymbol{K}_{(p, q)} / \mathbb{Q}(\sqrt{p})$, so that by the previous arguments, $l$ splits in $\boldsymbol{k}_{+}=\mathbb{Q}(\sqrt{p q})$. Let us note that a prime $l$ (with $l \neq p$ and $l \neq q)$ splits in $\mathbb{Q}(\sqrt{p q})$ if and only if $\left(\frac{p q}{l}\right)=+1$, hence if and only if $\left(\frac{l}{q}\right)=\left(\frac{p}{l}\right)$. We have thus proved:

Theorem 7. Let $l$ denote any prime. Let $p \not \equiv 3(\bmod 4)$ and $q \equiv 1$ $(\bmod 4)$ be two distinct primes such that $q$ splits in the real quadratic field $\mathbb{Q}(\sqrt{p})$. If the relative class number of a dihedral octic CM-field $\boldsymbol{N}_{(p, q)}$ is one, then we have:

(a) $\left(\frac{l}{q}\right)=-1$ if $\left(\frac{p}{l}\right)=-1$ and $4 \leq l^{2}<q / 16$;

(b) $\left(\frac{l}{q}\right)=+1$ if $\left(\frac{p}{l}\right)=+1$ and $2 \leq l<q / 16$.

Now, there are exactly 40 values of

$$
\Delta= \begin{cases}p q & \text { if } p \neq 2 \\ 8 q & \text { if } p=2\end{cases}
$$

with $2 \leq p<q$ two primes such that $p \not \equiv 3(\bmod 4), q \equiv 1(\bmod 4)$, $\left(\frac{p}{q}\right)=+1, \Delta<5 \cdot 10^{9}$ and (a) and (b) of Theorem 7 are satisfied. The largest one is $\Delta=113977$. Let us note that as we have assumed that $p<q$, 
these necessary conditions (a) and (b) imply

$$
\left(\frac{\Delta}{l}\right)=+1 \quad \text { for all primes } l \text { such that } l<\Delta^{1 / 4} / 4
$$

so that we can hope to get only few values of $\Delta$ less than $5 \cdot 10^{9}$ such that these conditions (a) and (b) are fulfilled. It is worth mentioning that if one wants to solve the class number one problem for $\boldsymbol{K}_{(p, q)}$ then one cannot assume that $p<q$ for we do not have $h\left(\boldsymbol{K}_{(p, q)}\right)=1 \Leftrightarrow h\left(\boldsymbol{K}_{(q, p)}\right)=1$, i.e. the symmetric relation $h^{*}\left(\boldsymbol{K}_{(p, q)}\right)=h^{*}\left(\boldsymbol{K}_{(q, p)}\right)$ does not always hold for the absolute class numbers. For example, we will note below that $h\left(\boldsymbol{K}_{(17,257)}\right)=$ $1 \neq 3=h\left(\boldsymbol{K}_{(257,17)}\right)$. But now, if $q$ is small then the necessary conditions of Theorem 7 are likely to be satisfied for many $\Delta=p q$ less than $5 \cdot 10^{9}$, so that if our criterion of Theorem 6 had been written as a necessary condition for the class number of a CM-field to be one, then it would have provided us with a lot of values of $\Delta=p q$ less than $5 \cdot 10^{9}$, so that it would have been difficult to solve the class number one problem for non-normal quartic CM-fields. This in fact motivated the authors to discuss everything in terms of relative class numbers instead of class numbers.

We would like to point out that there are several tricks in order to speed up the sieve based on the necessary conditions of Theorem 7 . Indeed, from (a) and (b) written with $l=2$ we see that $p \equiv q(\bmod 8)$ provided that $p$ and $q$ are odd primes such that $q>64$. Hence, $\Delta \equiv 1(\bmod 8)$ provided that $\Delta$ is odd and $\Delta>4096$. Moreover, if $p=2$, then $q \equiv 1(\bmod 8)$. Moreover, we can get much better lower bounds on $\Delta$ than the one given in Theorem 5 , provided that $p$ is a small prime. Indeed, we have:

TheOrem (see [Lou 1, Th. C]). Let $\boldsymbol{K}$ be a non-normal quartic CM-field with real quadratic subfield $\boldsymbol{k}$ such that $d(\boldsymbol{K}) \geq 16 d(\boldsymbol{k})^{2}$. Let $\varepsilon(\boldsymbol{k})>1$ and $h(\boldsymbol{k})$ be the fundamental unit and the class number of $\boldsymbol{k}$. If $h^{*}(\boldsymbol{K})=1$, then

$$
\left(1-\frac{13}{d(\boldsymbol{K})^{1 / 4}}\right) \frac{\sqrt{d(\boldsymbol{K})}}{\log (d(\boldsymbol{K}))} \leq 481 h(\boldsymbol{k}) \log (\varepsilon(\boldsymbol{k})) .
$$

Thus, for example, with $p=2$ we get $h^{*}\left(\boldsymbol{K}_{(2, q)}\right)>1$ provided that $d\left(\boldsymbol{K}_{(2, q)}\right)=64 q>8 \cdot 10^{7}$ (see (5) for the equality), i.e. provided that $\Delta=$ $8 q>10^{7}$, i.e. provided that $q>2 \cdot 10^{6}$.

Second, there are only 30 of these 40 values of $\Delta=p q$ such that the fundamental unit $\varepsilon_{p q}$ of the real quadratic field $\mathbb{Q}(\sqrt{p q})$ is such that $N_{\mathbb{Q}(\sqrt{p q}) / \mathbf{Q}}\left(\varepsilon_{p q}\right)=+1$. Let us point out that the class numbers of the corresponding real quadratic fields $\mathbb{Q}(\sqrt{p})$ are all equal to one. The class numbers $h_{p q}$ of the corresponding real quadratic fields $\mathbb{Q}(\sqrt{p q})$ are given in the following table: 


\begin{tabular}{|c|c|c|c|c|c|c|c|}
\hline$(p, q)$ & $h_{p q}$ & $(p, q)$ & $h_{p q}$ & $(p, q)$ & $h_{p q}$ & $(p, q)$ & $h_{p q}$ \\
\hline$(2,17)$ & 2 & $(5,41)$ & 2 & $(5,269)$ & 6 & $(41,73)$ & 6 \\
$(2,73)$ & 2 & $(13,17)$ & 2 & $(17,89)$ & 2 & $(29,149)$ & 10 \\
$(2,89)$ & 2 & $(5,61)$ & 2 & $(37,41)$ & 2 & $(17,257)$ & 2 \\
$(2,97)$ & 2 & $(13,29)$ & 2 & $(29,53)$ & 2 & $(73,97)$ & 2 \\
$(2,233)$ & 2 & $(5,101)$ & 4 & $(5,389)$ & 2 & $(97,313)$ & 2 \\
$(2,281)$ & 2 & $(5,109)$ & 2 & $(13,157)$ & 2 & $(109,421)$ & 2 \\
& & $(5,149)$ & 2 & $(17,137)$ & 2 & $(173,269)$ & 4 \\
& & $(13,61)$ & 4 & $(13,181)$ & 2 & $(193,337)$ & 4 \\
\hline
\end{tabular}

Third, only 26 out of these 30 values of $(p, q)$ are such that $\boldsymbol{K}_{(p, q)}$ is a non-normal quartic CM-field with odd relative class number, namely the ones such that 4 does not divide $h_{p q}$ (Theorem 4). Now, we sum up the numerical computation of the relative class numbers of the corresponding 26 non-normal quartic CM-fields $\boldsymbol{K}_{(p, q)}$ :

\begin{tabular}{|c|c|c|c|c|c|}
\hline$(p, q)$ & $h^{*}\left(\boldsymbol{K}_{(p, q)}\right)$ & $(p, q)$ & $h^{*}\left(\boldsymbol{K}_{(p, q)}\right)$ & $(p, q)$ & $h^{*}\left(\boldsymbol{K}_{(p, q)}\right)$ \\
\hline$(2,17)$ & 1 & $(5,41)$ & 1 & $(5,389)$ & 1 \\
$(2,73)$ & 1 & $(13,17)$ & 1 & $(13,157)$ & 1 \\
$(2,89)$ & 1 & $(5,61)$ & 1 & $(17,137)$ & 1 \\
$(2,97)$ & 3 & $(13,29)$ & 1 & $(13,181)$ & 1 \\
$(2,233)$ & 1 & $(5,109)$ & 1 & $(41,73)$ & 11 \\
$(2,281)$ & 1 & $(5,149)$ & 1 & $(29,149)$ & 5 \\
& & $(5,269)$ & 1 & $(17,257)$ & 1 \\
& & $(17,89)$ & 7 & $(73,97)$ & 1 \\
& & $(37,41)$ & 3 & $(97,313)$ & 17 \\
& & $(29,53)$ & 1 & $(109,421)$ & 11 \\
\hline
\end{tabular}

Let us point out that the first author computed these class numbers using the method developed in [Lou 2], [Lou 3] and the second author computed them using the method developed in $[\mathrm{Ok}]$. As the results agreed, we can be quite confident of them. Hence, we get:

TheOREM 8. There are exactly 19 non-isomorphic dihedral octic CMfields with relative class number one, namely the $\boldsymbol{N}_{(p, q)}$ 's with

$$
\begin{aligned}
(p, q) \in\{ & (2,17),(2,73),(2,89),(2,233),(2,281), \\
& (5,41),(5,61),(5,109),(5,149),(5,269),(5,389), \\
& (13,17),(13,29),(13,157),(13,181), \\
& (17,137),(17,257),(29,53),(73,97)\} .
\end{aligned}
$$


Hence, there are exactly 38 non-isomorphic non-normal quartic CM-fields with relative class number one, namely the $\boldsymbol{K}_{(p, q)}$ 's and $\boldsymbol{K}_{(q, p)}$ 's with $p$ and $q$ as above.

5. Determination of the non-normal quartic CM-fields and of the dihedral octic CM-fields with class number one. If we compute the class numbers of the real quadratic subfields of these 38 non-normal quartic CM-fields with relative class number one, we see that there exist 37 non-isomorphic non-normal quartic CM-fields with class number one, the exceptional field being $\boldsymbol{K}_{(257,17)}$ for which we have $3=h\left(\boldsymbol{K}_{(257,17)}\right) \neq$ $h\left(\boldsymbol{K}_{(17,257)}\right)=1$. Now, we compute the class numbers of the 18 dihedral octic CM-fields with relative class number one.

Lemma (see [Lou 4], [Kub]). Let $p \not \equiv 3(\bmod 4)$ and $q \not \equiv 3(\bmod 4)$ be two distinct primes such that the fundamental unit $\varepsilon_{p q}>1$ of the real quadratic field $\mathbb{Q}(\sqrt{p q})$ is such that $N_{\mathbb{Q}(\sqrt{p q}) / \mathbb{Q}}\left(\varepsilon_{p q}\right)=+1$. Then the class number $h\left(\boldsymbol{k}_{(p, q)}\right)$ of the totally real bicyclic biquadratic number field $\boldsymbol{k}_{(p, q)}=$ $\mathbb{Q}(\sqrt{p}, \sqrt{q})$ is equal to $\frac{1}{2} h_{p} h_{q} h_{p q}$ where $h_{d}$ stands for the class number of the real quadratic field $\mathbb{Q}(\sqrt{d})$.

Hence, exactly 17 of the 19 dihedral octic CM-fields with relative class number one have class number one too. The two dihedral octic CM-fields $\boldsymbol{N}_{(p, q)}$ with relative class number one but with class numbers greater than one are given in the following table:

\begin{tabular}{|c|c|c|}
\hline$(p, q, p q)$ & $\left(h_{p}, h_{q}, h_{p q}\right)$ & $h\left(\boldsymbol{N}_{(p, q)}\right)$ \\
\hline$(5,269,1345)$ & $(1,1,6)$ & 3 \\
$(17,257,4369)$ & $(1,3,2)$ & 3 \\
\hline
\end{tabular}

and we get:

THEOREM 9. (a) There are exactly 37 non-isomorphic non-normal quartic CM-fields with class number one, namely the $\boldsymbol{K}_{(p, q)}$ 's with

$$
\begin{aligned}
(p, q) \in\{ & (2,17),(2,73),(2,89),(2,233),(2,281), \\
& (5,41),(5,61),(5,109),(5,149),(5,269),(5,389), \\
& (13,17),(13,29),(13,157),(13,181), \\
& (17,2),(17,13),(17,137),(17,257), \\
& (29,13),(29,53),(41,5),(53,29), \\
& (61,5),(73,2),(73,97)(89,2),(97,73), \\
& (109,5),(137,17),(149,5),(157,13),(181,13), \\
& (233,2),(269,5),(281,2),(389,5)\} .
\end{aligned}
$$


(b) There are exactly 17 non-isomorphic dihedral octic CM-fields with class number one, namely the $\boldsymbol{N}_{(p, q)}$ 's with

$$
\begin{aligned}
(p, q) \in\{ & (2,17),(2,73),(2,89),(2,233),(2,281), \\
& (5,41),(5,61),(5,109),(5,149),(5,389), \\
& (13,17),(13,29),(13,157),(13,181), \\
& (17,137),(29,53),(73,97)\} .
\end{aligned}
$$

\section{References}

$[\mathrm{H}] \quad$ J. Hoffstein, Some analytic bounds for zeta functions and class numbers, Invent. Math. 55 (1979), 37-47.

[Kub] T. Kubota, Über die Beziehung der Klassenzahlen der Unterkörper des bizyklischen biquadratischen Zahlkörper, Nagoya Math. J. 6 (1953), 119-127.

[Lou 1] S. Louboutin, On the class number one problem for non-normal quartic CMfields, Tôhoku Math. J., to appear.

[Lou 2] -, Calcul du nombre de classes des corps de nombres, preprint, 1992.

[Lou 3] -, Calcul des nombres de classes relatifs. Application aux corps octiques quaternioniques à multiplication complexe, C. R. Acad. Sci. Paris Sér. I 317 (1993), 643-646.

[Lou 4] -, Determination of all quaternion octic CM-fields with class number 2, preprint, 1993.

[O] A. M. Odlyzko, Some analytic estimates of class numbers and discriminants, Invent. Math. 29 (1975), 275-286.

[Ok] R. Okazaki, On evaluation of L-functions over real quadratic fields, J. Math. Kyoto Univ. 31 (1991), 1125-1153.

[Se] J. P. Serre, Corps Locaux, Hermann, 1968.

[W] L. C. Washington, Introduction to Cyclotomic Fields, Graduate Texts in Math. 83, Springer, 1982.

[Y] K. Yamamura, The determination of the imaginary abelian number fields with class number one, Math. Comp., to appear.

UNIVERSITÉ DE CAEN, U.F.R. SCIENCES

DÉPARTEMENT DE MATHÉMATIQUES

ESPLANADE DE LA PAIX

14032 CAEN CEDEX, FRANCE

E-mail: LOUBOUTI@UNIV-CAEN.FR
DEPARTMENT OF MATHEMATICS

FACULTY OF SCIENCE KYOTO UNIVERSITY

KYOTO 606-01, JAPAN

E-mail: OKAZAKI@KUSM.KYOTO-U.AC.JP 